\title{
La idea educativa y sanitaria de Juan Luis Vives: un estudio gadameriano
}

\section{The educational and sanitary concept of Juan Luis Vives: A Gadamerian-hermeneutic study A idéia educativa e sanitária de Juan Luis Vives: Um Estudo Gadameriano}

\author{
Juana Hernández Conesa ${ }^{1}$. Enrique Maldonado Suarez ${ }^{2}$ \\ ${ }^{1}$ Profesora Titular de Universidad. Investigadora Principal del Grupo de Investigación EDUCACIÓN, Historia y ciencia en el \\ arte de la sanación (EHYCARS). Medalla Florence Nightingale. 2009. \\ ${ }^{2}$ Profesor Universidad Autónoma de Madrid. Facultad de Medicina. Miembro del Grupo de Investigación E063-06 EDUCA- \\ CIÓN, HISTORIA Y CIENCIA EN EL ARTE DE LA SANACIÓN (EHYCARS). \\ Cómo citar este artículo en edición digital: Hernández Conesa, J. y Maldonado Suárez, E. (2014) \\ La idea educativa y sanitaria de Juan Luis Vives: un estudio gadameriano. Cultura de los Cuidados (Edición digital) 18, 38. \\ Disponible en: http://dx.doi.org/10.7184/cuid.2014.38.11> \\ Correspondencia: Juana Hernández Conesa. Facultad de Enfermería. Universidad de Murcia. Campus de Espinardo. 30100 Murcia. \\ Correo electrónico: e-mail: jhconesa@um.es \\ Recibido: 21/11/2013; Aceptado: 16/01/2014 \\ Correo electrónico: irene.lopezleon@alum.uca.es
}

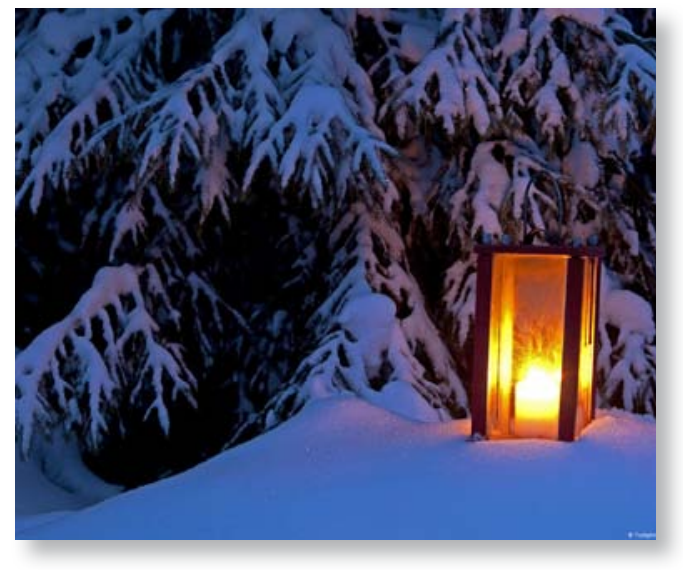

ABSTRACT

European fragmentation, due in part to the ratification of the Council of Trent, was a significant incident which allows a polyhedral analysis. From this moment, a new divergent and confronted way to rebuild the world was developed between "both Europes". This fact led to a two different mentalities preface from the braudelian perspective, co-existing under a continuous struggle. On one side, the North
European Countries, which descended from the Protestant Reformation. On the other side were the South European Countries, inheriting the Counter-reformation proper and the Tridentine postulates.

Our hypothetical proposal is derived from the educational and sanitary concept included in the work of Juan Luis Vives. Through its study it has been corroborated that, the European fragmentation had many consequences beyond the circumstantial time when it happened. In this research work, the source was studied following the hermeneutics analysis based on the Gadamerian interpretation.

Keyword: Juan Luis Vives. Education. Health. Council of Trent. Protestant Reformation

\section{RESUMO}

A Fragmentação da Europa, com uma ratificação em certa medida por parte do próprio Concílio de Trento, é um fato significativo que 
possibilita também análises poliédricos. Será a partir desse momento quando se produz um desenvolvimento progressivo entre as "duas Europas" com um modo discordado e enfrentado de construir o mundo. Observamos com tudo isso, os prolegômenos de duas mentalidades em sentido braudeliano, que continuarão vivendo uma luta continua. Europa do Norte: filha da Reforma Protestante e Europa do Sul: herdeira da Contrarreforma e da assunção, próprias das proposições tridentinas.

Subentendendo-se, é na idéia educativa e sanitária que é dada na obra de Juan Luis Vives onde surge nossa concepção hipotética e onde constatamos que essa separação trouxe consequências mais alem do tempo circunstancial o conjuntural onde aconteceu. Assim mesmo, de uma análise das fontes, para uma abordagem da investigação escolhemos uma estratégia metodológica de caráter hermenêutico, baseado no Giro de Gadamer(Gadamer 2007) .

Palavras-chave: Juan Luis Vives. Educação. Saúde. Concílio de Trento. Reforma Protestante.

\section{RESUMEN}

La fragmentación de Europa, tras la ratificación por una parte de ella del Concilio de Trento, es un hecho significativo que permite, a su vez, análisis poliédricos. Será desde ese momento donde se irá gestando entre las "dos Europas" un modo discrepante y enfrentado de construir el mundo. Asistimos, con este hecho, a los prolegómenos de dos mentalidades en el sentido braudeliano, que vivirán en una continua pugna. La Europa del Norte: hija de la Reforma Protestante y la Europa del Sur: heredera de la Contrarreforma y de la asunción, como propios, de los postulados tridentinos.

Si bien, es en la idea educativa y sanitaria que se desprende de la obra de Juan Luis Vives desde donde surge nuestro planteamiento hipotético y en donde hemos constatado que esa ruptura, tuvo consecuencias más allá del tiempo circunstancial o coyuntural donde aconteció. Asimismo, desde un análisis de las fuentes, para abordar la investigación hemos optado por una estrategia metodológica de carácter hermenéutico, basada en el Giro de Gadamer .

Palabras Clave: Juan Luis Vives. Educación. Sanidad. Concilio de Trento. Reforma Protestante.

Hubo un tiempo en Europa, antes de la segmentación, un tiempo fundacional, un tiempo que Nebrija aglutina de manera notable. Para los estudiosos las Introductiones latinae (Nebrija, 1481), "trajeron la modernidad a la lengua y la traza a la literatura" Porque, las Introductiones latinae eran un ofensiva al concepto del saber, a la idea del enseñar y del aprender, y, en definitiva, una invasión a la inaccesible y hermética escolástica, en la España Bajomedieval.

Nebrija proponía un instrumento, esencial y provechoso, que, aún siguiendo los principios de los clásicos, debía desalojar las sinuosidades en los planteamientos y explicaciones de las cuestiones. Era un propósito que dejaba atrás la oscuridad escolástica, y esta nueva idea de la lengua se funda, según Francisco Rico en la "experiencia, en la literatura y en la historia". De tal modo, que se pudiera estudiar y conocer a los grandes, a los clásicos, pero de una forma bien distinta. Conocer a los clásicos, insiste Nebrija, pero con criterio; criterio para discernir y advertir que algunos autores serían prescindibles y otros no. Distinguiendo en ellos, "época y logros".

Nebrija, inspirado en Quintiliano y Cicerón, urgía a la "claridad y a la naturalidad de la 
lengua. Y de este modo se crea un modelo de pensamiento, es decir: una forma distinta de plantear y resolver problemas". En definitiva, una didáctica. "Y ese modo de pensamiento es, nada más y nada menos, que el paradigma del humanismo renacentista".

Porque el retorno a la Antigüedad, pensada de otro modo, nos conduce, cuando menos, a una forma de enseñar y de aprender diferente, $y$, por ende, a una didáctica nueva. $Y$ es en esa invectiva cognitiva entre el ayer inmediato y su forma de entender el Conocimiento - en la Baja Edad Media-, y ese otro modo de aprehender el ayer remoto para explicar, renovar y construir el presente -el Renacimiento y el Humanismo-, donde se fundamenta, a nuestro juicio y a la luz de lo estudiado, la Pedagogía del Humanismo renacentista. Y, Nebrija, entre otros, está inspirado en una realidad crítica y objetora frente al inmovilismo escolástico. Nebrija, representa igualmente, una forma de magisterio nuevo, basado "en el prestigio de las fuentes antiguas o en los humanistas italianos y que continuará en Salamanca Sánchez de las Brozas" (Closa 1994)

Con todo, en aquel tiempo, emerge una nueva idea de formación que reorienta los fines, los métodos, los contenidos, los textos y las acciones didácticas. La renovación educativa aflora con una tenacidad enérgica. En este sentido la contribución de Vives es reconocida como sobresaliente por todos los tratadistas de la Historia de la Educación, que juzgan su determinación contra los modos escolásticos en In speudodialecticos o más tarde en De causis corruptarum artium o sus perspectivas de desarrollo en cuanto a los contenidos y a los modos de exponerlos que se congregan en el De tradendis disciplinis.

De tal manera, y a la luz de lo estudiado, entendemos que en la pedagogía vivesiana, se enuncian los rasgos de la modernidad: sencillez y rigor intelectual, apelación a la razón clara y a la observación metódica, sentido instrumental de la lengua, y en particular a la vernácula, para aproximarse al vinculo que existe entre la vida intelectual, la experiencia y la vida práctica. En Vives se convocan al tiempo, a través de lo formativo, la rectitud moral y la utilidad social: un interés ético-político que subraya la preocupación civil del Humanismo.

Igualmente, las orientaciones metodológicas o principios pedagógicos de actuación que se fundamentan en su advertido análisis psicológico y en la precisa tipificación de las causas que pervierten la enseñanza, constituyen, a nuestro juicio, también y sobre todo, una apreciable aportación al entramado educativo de la Edad Moderna.

La obra de Vives viene a pronunciarse en palabras de Mayodormo (Mayordomo 1992) "sobre un tema en el que, no mucho después, otro español - Huarte de San Juan- dedicaría una atención especial con notable éxito en influjo: conducir la educación desde una cuidada observación del desarrollo psicológico del alumno; se trata, en definitiva de conocer el "ingenio" de aquél para orientar el proceso según sus aptitudes. Vives auspicia el nacimiento, en el ideario pedagógico moderno, de una pedagogía diferencial, se anticipa al llamado " realismo pedagógico", haciendo considerar el interés de tener en cuenta la diversidad de capacidades existentes y la pluralidad de tratamientos que ellos conlleva".

La vasta glosa didáctica en la obra de Vives se completa con logradas correspondencias entre la corrupción de las disciplinas y el modo de enseñar. Hemos seleccionado algunos fragmentos acerca de lo que nos dice acerca del trabajo, de la memoria y de los maestros. $Y$ con el método gadameriano (Hernández, 2012), 
propuesto para este estudio, hemos relacionado concepto y palabra y por ende, explicado pensamiento y lenguaje para, de este modo, establecer las relaciones conceptuales como espacios significativos de explicación.

[...] y en tanto que Dios no otorga sus dones a los ociosos, precisamente por ello has de obrar con diligencia y afán en la afición por las letras y la virtud [...] (Mayordomo, 1992). [...] Has de saber que la memoria es el tesoro de toda erudición, y si falla, todo esfuerzo es baldío, no otra manera que si viertes agua en un cesto. Pero a nadie le ha correspondido una memoria tan infeliz que no pueda convertirse en felicísima con el ejercicio. No hay nada que se goce más el trabajo y el esfuerzo. Como que tampoco hay nada que se corrompa y parezca a causa de la actividad y el ocio. Por ello se ha de aprender cada día algo, aun cuando no haya necesidad, aunque sólo fuera para que no invada la memoria una modorra, enfermedad perniciosa como no hay otra. Para conservar la memoria en óptimas condiciones, lo primero que hemos de evitar es la gula, el hartazgo, la indigestión, la crápula, el vino desmesurado, y la excesiva cerveza, así como dormir boca arriba [...].

Vives, exige capacidad a los maestros; esfuerzo y honestidad, también, como a los estudiantes; propone acerca del método y demanda que el aprendizaje se produzca por grados; como argumento donde se asientan los pilares de la Psicología del Aprendizaje, que él mismo inaugura.

[...] Has de amar, venerar y respetar a tu maestro no menos que a tu padre. Cosa cierta es que los preceptores nos ofrecen como un retrato de nuestros padres, y de nadie puedes recibir un servicio mayor que de en la vida con estos dos bienes. Añade a ello que aprenderás más fácilmente si aprecias a quien te enseña y si no desprecias o descuidas nunca sus dictados. Recíbelos con respeto en tu pecho tenlos como oráculos. Y no sólo has de amarlo, sino que debes procurar con todo tu empeño ser correspondido por él; así te enseñará con mayor diligencia. Su aprecio lo conseguirás siguiendo con modestia sus preceptos, si lo respetas y honras, así como admirando todo aquello que hiciera o dijera. Si aprueba algún determinado aspecto de la vida o del discurso, obra de modo que se percate de que tú también lo apruebas; si reprueba algo, evítalo. Escúchalo atento: toma buen nota de sus palabras, de sus modismos, sus sentencias, y a través de la imitación modélate semejante a él, en la medida de tus fuerzas. Cuando el preceptor se dé cuenta de ello se esforzará por su parte para que no puedas recibir nada suyo que fuera indigno de emulación [...].

Para constatar nuestra hipótesis en este estudio hemos de atender a lo que Vives, en el Prefacio a los libros de las disciplinas (Riber, 1948) pone de manifiesto, poniendo de manifiesto su disconformidad con Aristóteles y su idea acerca de la crítica frente a la ciega autoridad, insistiendo en la importancia de la experiencia. En estos fragmentos se advierte la diferencia existente entre la propuesta vivesiana y el ideario pedagógico de la España de la Contrarreforma: la Ratio Studiorum:

[...] por atreverme a discrepar de unos escritores consagrados por los siglos, y singularmente de Aristóteles [...]

[...] no cabe duda que es mucho más conveniente para el progreso de la cultura aplicar la crítica a los escritos de los grandes autores, que descansar perezosamente en la sola autoridad y aceptar sistemáticamente todo cuanto nos proporciona la fe ajena [...]

[...] ¡Cuán ancha puerta de acceso a todas las disciplinas nos abren los descubrimientos 
de los siglos anteriores y una tan continuada experiencia! [...].

[...] como dice Séneca con su habitual agudeza y discreción: Aquellos que antes que nosotros promovieron esos estudios, no son nuestros amos, sino nuestros guías. La verdad es accesible a todos y no está aún ocupada completamente. Muy mucha parte de ella quedó reservada a los venideros [...].

Finalmente, se hace notar que estamos ante una nueva forma de abordar el Conocimiento: para todos aquellos que sean estudiosos, honorables y se forjen en y con la experiencia. Y, también, estamos ante un distinto modo de enseñarlo.

Para abordar la idea sanitaria de Vives, en este estudio, nos hemos ceñido a la obra De Subventione pauperom (Vives, 1526), escrita en Flandes, en la cuidad de Brujas y dedicada a los Cónsules y Senado de dicha ciudad. Su primera edición data de marzo de 1526. Si bien, como apunta Joaquín Parellada (Parellada, 1998), existe una traducción al español, inédita, de Bernardo Pérez de Chinchón, que el propio Parellada descubrió y cuyo manuscrito se conserva en Valencia. Manuscrito, que Parellada (Maestre, 2002) da como autógrafo, con toda probabilidad. Sólo Pons Fuster (Pons, 1995) había mencionado esta conexión de Bernardo Pérez con Vives. Sin embargo, es bien conocido que su protector principal fue el tercer Duque de Gandía (Jiménez, 1978): Don Juan de Borja, padre de San Francisco.

La traducción hecha por el canónigo Pérez de Chinchón De Subventione pauperom, según Matheeussen (Matheeussen, 1986) es de la primera edición de 1526. Ya que en la segunda, en septiembre de ese mismo año, Vives añade nuevos párrafos. No obstante, lo que sí declara el hallazgo de Parellada es el anhelo por traducir la obra, así como la estrecha corres-

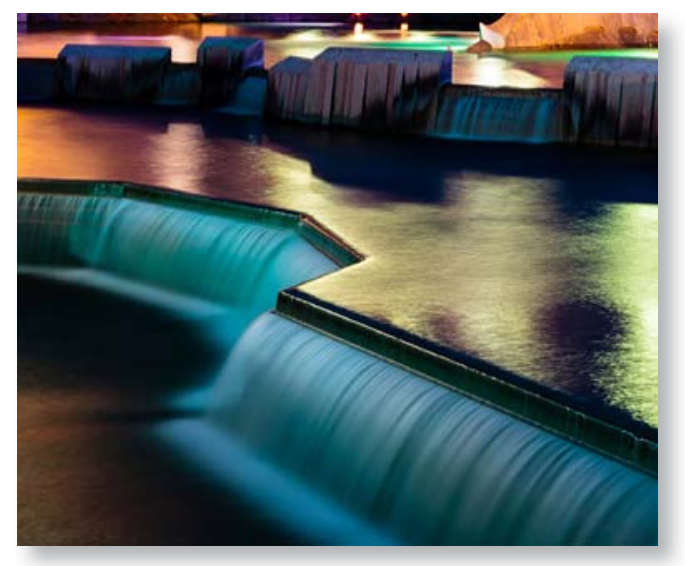

pondencia que mantenía Pérez de Chinchón, con Vives. Sin duda, estas revelaciones nos presentan al canónigo-traductor como un humanista cristiano sumamente comprometido con el Humanismo y que, asimismo, buscaba soluciones a los acuciantes problemas de la España de aquel entonces, la España del Emperador. Soluciones a las necesidades de los desfavorecidos, pobres, y por pobres-enfermos, que no tenían un lugar a donde acudir a curar sus buvas cuando reverdecen por no haberse curado bien. Y, entendemos, que el traductor Pérez de Chinchón, pretendía hallar algunas soluciones a esas apremiantes necesidades, traduciendo el De Subventione pauperom, de su compatriota y paisano Juan Luis Vives.

Para nuestro propósito las investigaciones en relación con la traducción del texto que ha realizado Parellada (Parellada, 1998) son sumamente significativas, ya que nos permite señalar matices en la intención del traductor, que están muy afectadas en otras traducciones, como la de Nieto Ibarra (Nieto, 1781). Es en ese sentido al acudir al hallazgo de $\mathrm{Pa}$ rellada, e introducir la intención de Pérez de Chinchón, advertimos que éste nos dibuja los asuntos sociales, como coetáneo, asuntos sociales a los que era sensible y que intentaba remediar; creando conciencia colectiva sobre los mismos. Y hallamos que apela, no sólo a la 
Caridad, sino a la responsabilidad de las autoridades civiles, a través del texto de Vives.

Vives se refiere a los trabajos que pueden desempeñar los ciegos, tanto hombres como mujeres:

[...] otros ciegos se den a la música, o aprendan a cantar a tañer cuerda, tecla, flauta; otros traygan tornos o cuerdas, según fuere el officio; otros traygan los fuelles en las fraguas, texan çestillas, canastillos, jaulas; hagan casas u otras cosillas de esta manera, que puedan bien aprender. Las mujeres ciegas hilen, devanen, hagan que quieran con tal que no stén ociosas [...] (Maestre, 2002).

Hemos escogido este fragmento ya que en él se confina, a nuestro juicio, un modelo social antagónico al que nos muestra el autor anónimo del Lazarillo y que entendemos pervivirá en la zona Contrarreformista de Europa. En el fragmento se informa que, en lugar de mendigar a cambio de rezos, al ciego se le debe inculcar la idea del trabajo y del esfuerzo, la idea de aprender un oficio, que le proporcione sustento digno y lo aleje de la pobreza, de las enfermedades, y de la miseria; en definitiva: de mendigar. Desarrollando, asimismo, sus capacidades y siendo útil a la sociedad. La diferencia. con respecto al personaje del ciego en el Lazarillo, es notable. Y nos desvela la génesis de lo que serán dos mentalidades enfrentadas.

En relación a las mujeres, a sus actitudes y competencias, Vives, no las deja al margen de cualquier acceso al Conocimiento, reclamando para ellas, recato y virtud. Si bien, se hace necesario sumergirnos en la mentalidad de la época y en el no menos importante libro que, alejado de los de caballería y piedad, circulaba en España en aquel tiempo: La Celestina ( $\mathrm{Pa}-$ rellada, 1998). De este modo, estaremos más próximos a comprender el registro socio-mo- ral en el que es traducida la obra de Vives. Una sociedad que hace ostensible su degradación moral en todos los estratos que la conforman. Una sociedad de "mendigos, amos, criados y pícaros", de "hidalgos venidos a menos" de "clérigos y aguaciles corruptos" de "impudor religioso" " de arribistas" y una sociedad atada por ley al Mayorazgo. Con todo, una sociedad con escasos planteamientos acerca del bien común y de la responsabilidad de los poderes públicos.

[Las muchachas] aprendan buenas opiniones y buena religión. Item a hilar, coser, texer, labrar, cocinar y desemboluer la casa; juntamente con esto aprendan templança, criança,vergüença, y sobre todo a ser castas, teniendo esto por el mas excellente don de las mugeres $[. .$.

También se hace necesario para nuestro propósito, y siguiendo a Parellada, hacernos eco de las alusiones a las profesiones marginales que se hallan en De Subventione pauperom, de Vives: los bordoneros y los verdugos y los que torturan. Acerca de los "bordoneros", es decir, según Covarruvias (Covarrubias, 1611) “aquellos que dissimulados con el ábito de peregrino y el bordón andan vagando por el mundo por no trabajar".

[...] los bordoneros, por ser bordoneros, tiene muchas veces mas sobervioso espíritu, que los ricos con quanto tienen. Aborreçen a todos a todos los que no les dan, o [no] los reprehenden [...]

Existe en la obra De Subventione pauperom, una prohibición imperiosa a la ociosidad, que es considera como la fuente de todas las desgracias, tanto para los pobres como para los ricos. Y están, igualmente, censurados los vicios a lo largo de todo el texto. De mismo modo, expone los peligros de la prostitución y de los que acuden a ella. 
[...] Dar al jugador, al putañero, no es otra cosa sino añadir stopa al fuego [...] (Maestre, 2002)

Entendemos que la crítica, que se advierte en la obra, respecto a los gastos de los ricos y del municipio, es de sumo interés para reflexionar acerca de los prolegómenos del modelo educativo y sanitario tridentino. Así como de las causas que constituyeron su financiación, estructura y finalidad, entre otros. Ya que más allá de los dictámenes del Concilio de Trento, es un modelo que no surge ex nihilo, emerge en el seno social que le precede y entendemos que emerge de ese modo, y no de otro, para paliar la ausencia de tales previsiones.

[...] Entonçes la ciudad deve de acordar algo en sus públicos gastos, como en lo que gasta en fiestas, juegos, toros, banquetes, presentes, regalos, y en processiones y auctos; todo esto mas tyra a vanagloria que no ha provecho [...].

Del mismo modo, Vives, alude al comercio $\mathrm{y}$ al dinero:

[...] A esto ha dado ocasión aver mucho dinero en que untar bien las manos [...]

Hemos de considerar que el dinero fluía venido de América. Y que en este "ir y venir", fueron muchos los que se hicieron con un patrimonio nada desdeñable. Sirva de entre las referencias que hemos consultado las que nos informan acerca de un verdadero aumento del precio de los terrenos en Valladolid, para la construcción de viviendas (Rico, 2006).

En cuanto a las necesidades básicas: la comida y el abrigo están presentes a lo largo de toda la obra.

[...] Contra el frío nos armamos de paño y afforros y contra el calor nos acojemos a las sombras [...] (Maestre, 2002).

En el Libro Primero, de la traducción de Nieto Ibarra, nos habla Vives del alimento como una medicina para fortalecer el cuerpo y nos dice que unos alimentos nos los proporciona la tierra y que otros los tomamos de ella, pero que son imprescindibles para vivir. Nos indica que el cuerpo, miserable y enfermizo debe ser ayudado por los remedios buscados a costa de la experiencia y que el ánimo afligido se aliviase con las conversaciones y obsequios de los amigos.

También nos indica que hay quienes escogieron a algunos de sus hijos de los mismos mendigos para enseñarles e instruirles en el modo de ganar la vida, les adoptaron por hijos dejándoles herederos en el Testamento[...] [...] Y ya que el mismo asunto nos ha puesto delante de los mendigos, si alguno considera su vida y vicios, y las atrocidades y delitos, que nos ofrecen cada día, se admirará más aun de quien haya quien los mire; tan perdido que da lo que se les da! [...].

Igualmente manifiesta su opinión acerca del lujo, de la virtud, la caridad y, de nuevo, del dinero que, al principio, fue como un medio para adquirir el sustento y vestido, pasó a ser instrumento universal del honor, dignidad, sobervia, ira, profusión, venganza, vida, muerte, imperio, en fin de todas las cosas que medimos por el dinero; subido su precio a un grado tan alto, nadie hay que no juzgue que se han de hacer diligencias para adquirirlo y conservarlo por todos los medios y caminos posibles, con razón o sin ella, justa o injustamente, y sin distinción de profano o sagrado, licito o ilícito; el que lo adquirió es tenido ya por sabio, Señor, Rey, hombre de grande y admirable consejo y talento; mas el pobre es reputado por necio, despreciable, y apenas por hombre; [...].

En el Libro Segundo, de la traducción de Nieto Ibarra, Vives alude, sin reservas, a la responsabilidad y conveniencia de que sean los Governadores de la Republica los que cuiden 


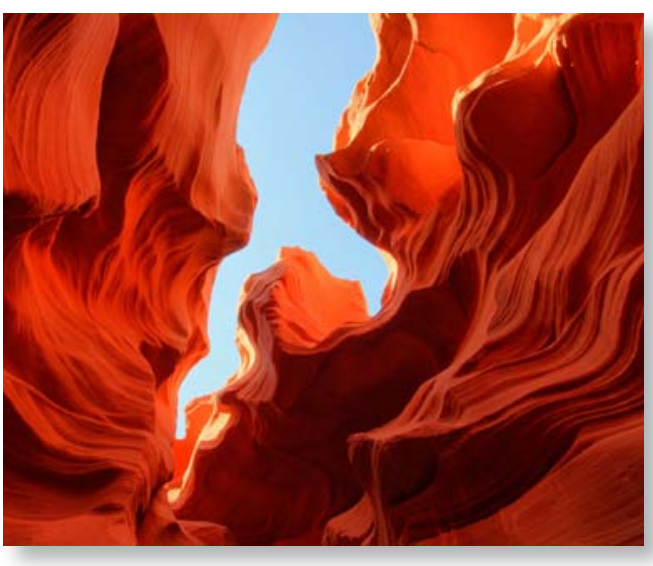

de los pobres. Y nos describe lo que, para él, es un hospital en aquellos años de la primera mitad del Quinientos, y que la gestión de los mismos y su mantenimiento pertenece a los gobernantes. Hecho éste que contrasta con la gestión, por parte de la Iglesia Tridentina, de los hospitales en la Europa Contrarreformista.

$[\ldots$.$] entre los pobres hay unos que viven en$ las casas comunmente llamadas hospitales, en griego Ptochotrofios, pero usaremos del primer vocablo como más conocido; otros mendigan publicamente; $y$ otros sufren como pueden cada uno en su casa sus necesidades: llamo hospitales aquellas casas en que se alimentan y cuidan enfermos, en que se sustenta un cierto número de necesitados, se educan los niños y niñas, se crian los expositos, se encierran los locos, y pasan su vida los ciegos: sepan los que goviernan la Ciudad que todo esto pertenece a su cuidado; [...].

Como podemos observar en el fragmento extraído del texto de Vives, los prolegómenos del modelo, tanto educativo como sanitario, pretridentino apuntaban en una dirección bien distinta al que los acontecimientos decretaron para el porvenir. Se evidencia, en Vives, un modelo sanitario gestionado desde los poderes civiles, con total sentido de la responsabilidad de éstos para con los ciudadanos y de los ciudadanos para con los que los gobiernan; se exhorta al esfuerzo y al trabajo desde una ideología humanista y cristiana. Apela, asimismo, el establecimiento de una red de hospitales públicos.

[...] todas aquellas obras que fuere necesario hacer en los hospitales, para que los caudales o rentas que dese el principio se dieron a los pobres, se consuman entre los pobres [...].

Juan Luis Vives, considera el trabajo como la virtud más elevada, y el ocio la madre de las desgracias de los hombres y de las ciudades, considera, asimismo, necesario el juicio de los médicos contra la picaresca de hacerse pasar por enfermo.

[...] Ante todas cosas se ha de decretar lo que impuso el Señor a todo el genero humano como por pena y multa del delito, es saber, que cada uno coma el pan adquirido con su sudor y trabajo $[\ldots]$.

[...] por eso no debe permitirse que viva alguno ocioso en la Ciudad, en donde como en una casa bien governada conviene que cada qual tenga su oficio [...].

[...] Se ha de tener consideración con la edad y quebranto de la salud, pero con la precaución de que no nos engañen con la ficcion o pretexto del achaque o enfermedad, lo que acontece no pocas veces; para evitar esto se recurrirá al juicio de los Medicos, castigando al que engañe [...].

A propósito de las personas que deben trabajar en los hospitales nos avisa y expresa que:

[...] atiendan al servicio de los enfermos, semejantes a aquellas viudas del principio de la Iglesia que tanto alababan los Apostoles; y en el tiempo que les quedare, hagan oración, lean, hilen, texan, y ocupense en alguna obra buena y honesta, como aun a las más opulentas y nobles Matronas manda San Geronimo [...].

En lo que respecta al cuidado de los niños subrayamos la concordancia de sus ideales sa- 
nitarios y educativos. Ambos: sistemas públicos y para todos. Nos expresa que las escuelas deben ser también para niñas en donde se les ha de enseñar las primeras letras. En esta solicitud se adelantó a todos los pedagogos reformista, en los que sin duda influyó. En oposición al sistema educativo y sanitario Contrarreformista que no la consideró.

[...] lo mismo digo de la escuela de niñas, en donde se han de enseñar los rudimentos de las primeras letras, y si alguna fuere apta y entregada al estudio, permitasele dilatarse en esto algo mas de tiempo [...].

En definitiva, el modelo sanitario al que nos alienta Vives en De Subventione pauperom, es un modelo sanitario que pretende ser solidario, laico, universal y público, sostenido con el peculio de los ciudadanos y administrado por los gobernantes $y$, por ende, supeditado a inspecciones.

[...] Si estos, Abades, y otros Superiores Eclesiasticos quisieran, aliviarian una grandisima parte de los necesitados con la grandeza de sus rentas [...].

[...] Hagase pues un computo de las rentas anuales de los hospitales, $\mathrm{u}$ hospicios [...].

[...] Nombre el Govierno dos Procuradores a cada hospital [...] [...] den estos todos los años cuenta de su administracion, y si agrada y se aprueba su fidelidad, continueseles en el cargo, sino, elijanse nuevos [...].

Con todo, al leer, de nuevo, la obra de Vives, y someterla a la interpretación gadameriana con el objeto que pretende esta investigación, hemos advertido su esclarecido, crítico, actual y perspicaz pensamiento. Que se manifiesta como antagónico al modelo sanitario tridentino.

En conclusión el modelo educativo y sanitario tridentino, según lo estudiado (Hernandez Conesa, 2013), no participó de la esencia pedagógica del Humanismo plasmada en la obra de Juan Luis Vives. Así, en los aspectos sanitarios será la Iglesia de Roma quien se haga cargo de los enfermos, pobres y desvalidos, fundamentalmente a través de las Órdenes Religiosas con Voto de Hospitalidad, sustituyendo al Estado en esta función social, tal y como propugna Vives. En lo relativo a la construcción pedagógica tridentina, ésta también queda bajo tutela eclesiástica, configurándose un analfabetismo de mentalidad, ya que no era necesaria la lectura de la Sagrada Escritura para ser un buen cristiano, a diferencia de la Europa de la Reforma. Si bien, es paradigmática la Ratio Studiorum de la Societatis Iesu, ya que en este sentido no albergamos duda alguna acerca de que el Humanismo era el frontispicio del templo educativo jesuítico. Sin embargo, la concepción teleológica de esta notable idea educativa: la Teología Tridentina, la aleja de la naturaleza del decurso formativo que pretendía unir lo intelectual, la experiencia y la vida práctica.

Finalmente lacraremos este estudio acerca de las "dos Europas" y de sus ideas educativas y sanitarias con una frase de Vives, que deseamos sea aplicada para con nuestras personas y para con nuestro trabajo:

Si en algún punto, amigos míos, os pareciere atinado mi parecer, sostenedlo por verdadero, no por mío.

\section{BIBLIOGRAFÍA}

- Anónimo. (1970) Lazarillo de Tormes, Edición de Francisco Rico. Salvat, Madrid.

- Closa Farré, J. (1994) Nebrija y el legado gramatical del Medievo. En Codoñer C, González Iglesias J.A. (ed.). Actas del Coloquio Antonio de Nebrija. Salamanca, Universidad de Salamanca.

- Covarrubias Orozco, S. (1611). Tesoro de la lengua castellana, o española. Imprenta Sanchez, Madrid. 
- Gadamer, H.G. (2007) El giro hermenéutico. Ed. Cátedra, Madrid.

- Gadamer, H.G. (2007) Verdad y Método. Ed. Sígueme, Salamanca.

- Hernández, J.M. Beneit, J.M. (2012) Un Estudio de los Paradigmas Educativos Enfermeros durante el Siglo XIX. Diego Marín Editor, Murcia.

- Hernández, JM Beneit, J.M. Maldonado, E \& Navarro, MA. (2013) El modelo educativo y sanitario de la España tridentina: entre la Auctoritas y la Potestas, Diego Marín Editor, Murcia.

- Maestre, J.M. Barea, J.P., \& Brea, L.C. (Eds.). (2002). Humanismo y pervivencia del mundo clásico: Homenaje al profesor Antonio Fontán. III. Editorial CSIC-CSIC Press, Madrid.

- Matheeussen, C. (1986) Quelques remarques sur le De subventione pauperum, en Ijswijn, J. y Losada, A. (eds), Erasmus in Hispania, Vives in Belgio. Peeters, Lovaina: 87-97.

- Mayordomo, A. (1992) Juan Luis Vives. Antología de textos. Estudis sobre la Valencia: Universidad de Valencia. ed., Vice-rectorat de Relacions Exteriors.

- Nebrija, E.A. (1481) Introductiones latinaes. Salamanca.
- Parellada, J. (1998) Una traducción inédita de Bernardo Pérez de Chinchón: "El Tratado llamado Socorro de Pobres", de Juan Luis Vives. Voz y Letra IX: 75-79.

- Pons Fuster, F. (1995) El mecenazgo cultural de los Borja de Gandía, Estudis 21: 23-44.

- Rico, F. (1996) Nebrija y la introducción del Renacimiento en España. Lección y Herencia de Elio Antonio de Nebrija; 1481-1981. En Actas de la III Academia Literaria Renacentista, 9-11 de diciembre de 1981. $1^{\text {a }}$ edición. Salamanca: Víctor García de la Concha.

- Riber, L. (1948) Juan Luis Vives. Obras completas. Primera Traslación castellana integra y directa, comentarios, notas y un ensayo bibliográfico. Vol. II. Aguilar, Madrid.

- Vives, J.L. (1978) Epistolario, editado por José Jiménez Delgado, Editora Nacional, Madrid.

- Vives, JL. (1526) De subventione pauperum sive de humanis necesitabus Libri II. Tip Huberti de Crook, Brugis.

- Vives, J.L. (1781) Tratado del Socorro de los pobres. Traducción de Juan de Gonzalo Nieto Ibarra. Imprenta de Benito Monfort. Valencia. 


\section{ANEXO FUENTES PRIMARIAS}

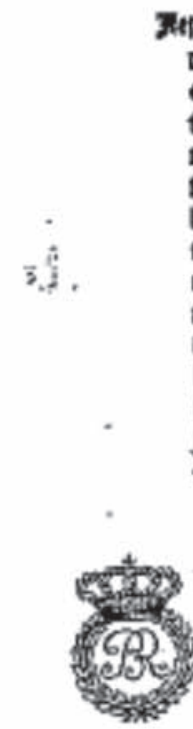

Brtius 3 Intenius lsbritefi. petro open

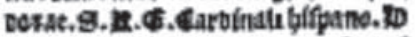

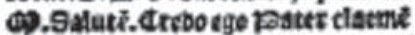
thifrme ateq optame non otfuturos quif me teneritaho et impuoentise acre fent:quoo rent ab anthquis et funfott bus grimathas betricam:et in quafü mastip futisua bominus ingerifa âa runt aufue fim quofin noutrm alrquob

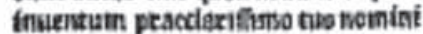
ostocare. Bullewe in frice mst opetts Iocenhore tptitola uallît teiponbere:tí a haec met orfputratto te hubice baben

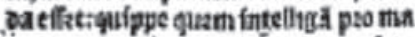
gnituzotre abiini atce ingenii prazican sanha nō mulths abmoba telectat1-3

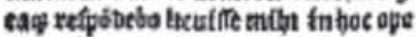

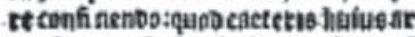

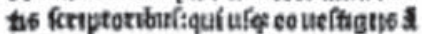
bquor inftuternint: er quod eft in pro

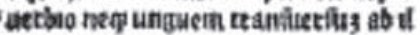

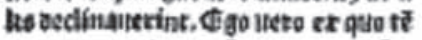

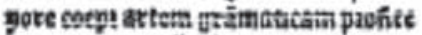
rtnunquast oiflaul cogutare-nues au ctorse ebikenbos imit 300 of abuffect

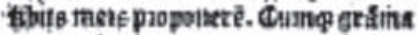
the quintolano suctore ouabebabeas pattzo:alceram pratenptenor quat aie thoonte:Aleträ frtitiño quac biftornce appedlatur: anbac fecunbainfinith po

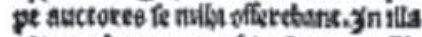

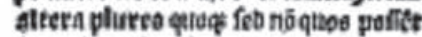
ingenta noftri faccult petônuere pue rorum mitefine nulbus baec Docerina parata eft. 2Dami curs illo feccuto pri ts lature fatent quats latund fermonits

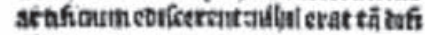
ale ques non perope thasn a zubstuo tettersmum polite. 1008 ueto quí plan mem a fermone Latulio zeficuimule: no

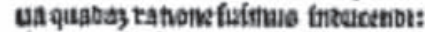
quatn multh fuptrioti astate multhe th

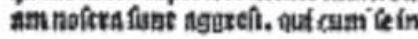

prinaptofitstusn operain tasimtenta puerte extetbeturoo polltecentut poif paucules uetue in rum confuftonis la bptinthuth fakbunt:untoe nec tyf fe facle extricare pofinnt. Filuuero ita a

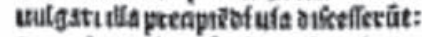
ouzuolunt uiberifanles et beruiratio ftubtof ut atiub qufeoam quam quoo

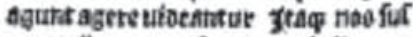
cepta tlloxnm petfone quoe infthtuste pofitemer: vilbel ferepánats naob no

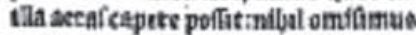
ex ne quiac abintrobuctiones latuma

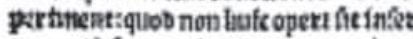
מam: nabeliquae alininbe mutuanta funt.

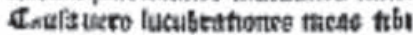
Debicantr Darrr aniplafirme fuit fur

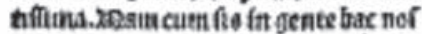
rea nó modo factorunt antríteo yeri: enas eiug botuatù artaum prefee ct pe ctlaareo quatsam nutela:fpero me is

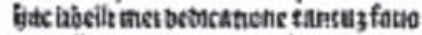
ne affequuthuram:ut ff albem lapilis:

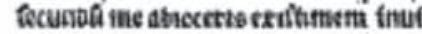
208 et dettractares meos inon poffectif sectum meut fufhnere. Eecte igttut quấ fyacas culmoe ceterı at'p vuas ptrcoces libero gntm ãphtuถ feturtan noftri laberne et ftugu; per

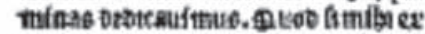
rio mitu osliturlature Hinguale boftes fuperare: quilus in acoithone bofus op to tetum intur quas bercuh mareres nofta expebthoutsy histapeuti ex ma nubins praebe boithts tabiocatise pe: folyenuo. suste tam firent facsas: né otatho crefereno in capert upers bre uitat et äplafitne cugnationi tue fit onerofs. vale. 

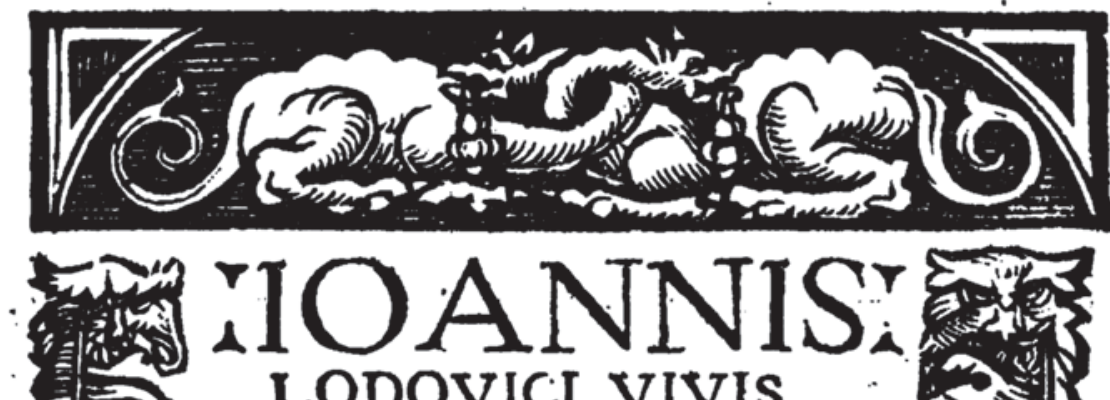

iI

OA

ANN

LODOVICI VIVIS VALENTINI

De fubuentione pauperú. Siue de

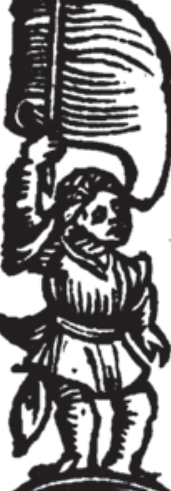
humanis neceffitatib' Libri .II. Ad Senatum brugenfem.

Prior de fubuetione priuata quid vnúquemq facere oporteat

ses

Alter de fubuentione publica, gd

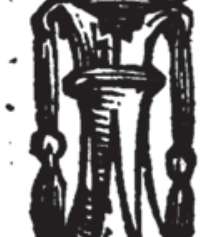
ciuitatem deceat. Ab autore ipfo recogniti.

Additę funt annotaciülę incalce

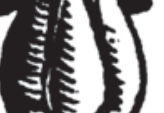
libri, ad explicandos aliquot diffi ciliores locos. Habes etiam indicé in fine.
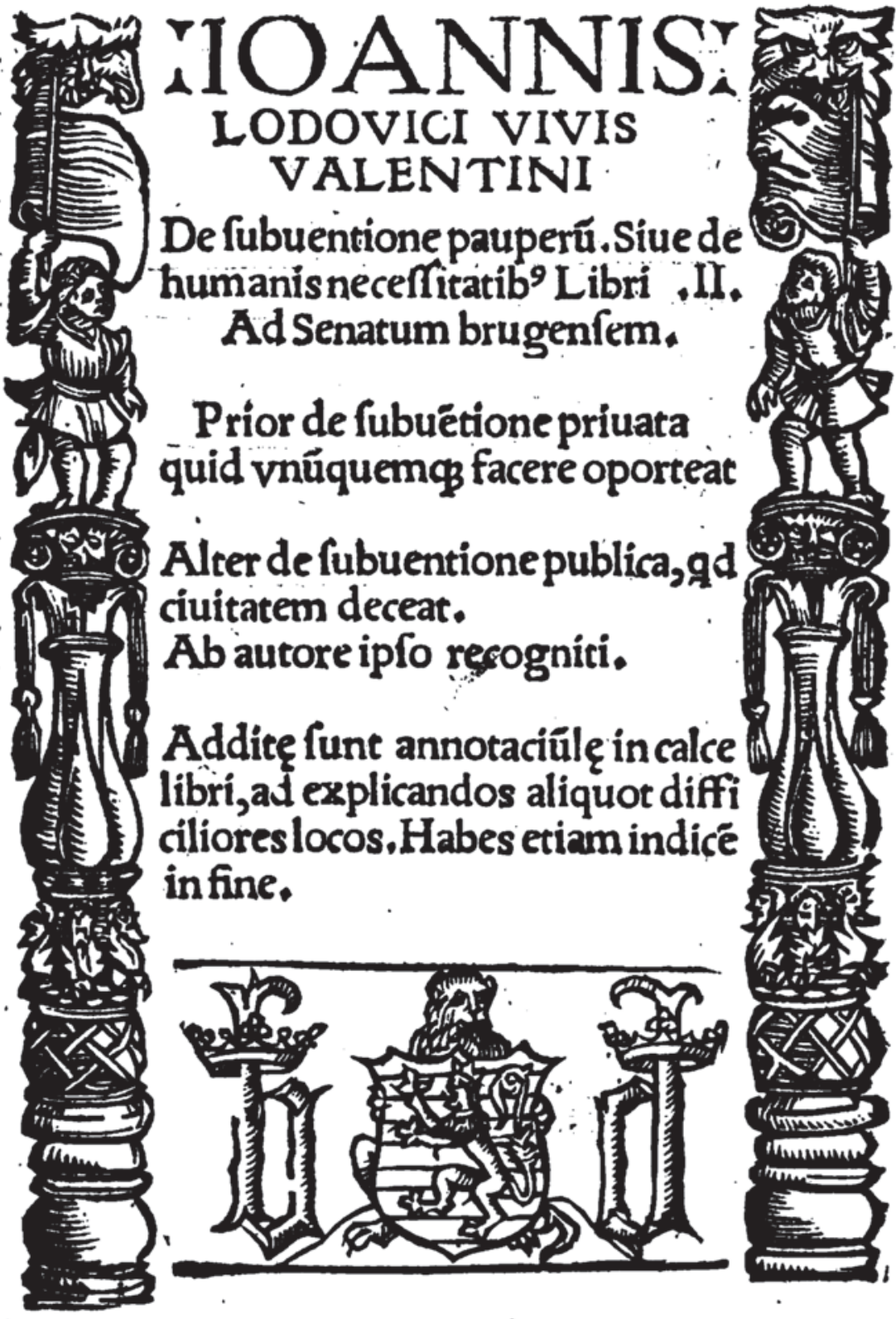

(iv)
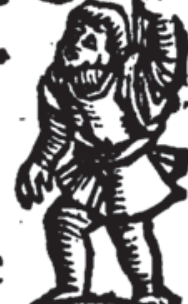

(1)
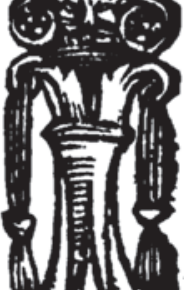
in

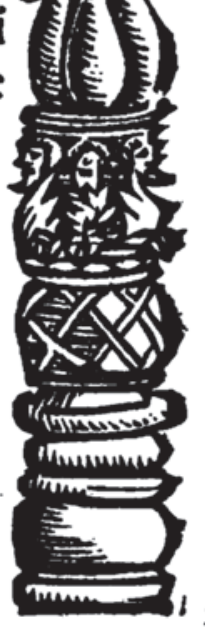




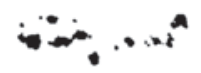

Ioannes Lodouicus Viucs

TOANNES LODOVICVS

IVIVES Confulibus \& Senatui

Brugenfi SALVTEM.

PEregrini \&aduenze officiú effe, tradic

I Cicero, in alíena republica nó eff́e curí ofum. Ita eft, nam in resalienascuriofitas vbige eft odiofa.cura $\&$ amica admonitio nō improbat̃. Quäğ neģ naturę lex alie nú ab hoie quicö finit effe, quod fit hoim. \&.Chrifti gratia velut tenaciff. glutinum hoies oés inter fe copulauit. Et ve fit alies

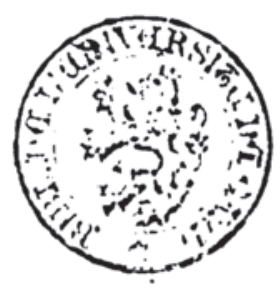
nú algd, ego vero fíc inciultaté hanc affi is cior, vt in Valentiă meam, nec àliter hanc nomino ğ patriá, cuiusiam quattuordeci annis fum incola, etfi tö cötinenter, femp tamé huc fum folit ${ }^{9}$ rediretanğ ad domû. Placuit ratioadminif ratiōis veftræ, eduo catio acciuilitas populi huius, \& incredibi lisin eo quies ac iufticia per gétescelebra, ta.Itaq \& vxoré hic duxi, nec fecus huic

- ciuitaticófultú veli, ğ ei, in qua, quod vitę reliquú Chriftibenigniras fecerit, traduce

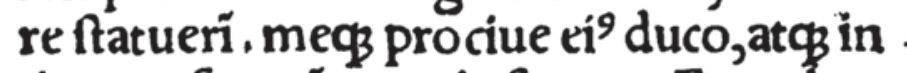
ciues eo fum aio quo in fratres. Etmulto

- rum exillisindigetix ad fribendú me im pulerunt, quéadmodã poffé eis fubueniri cenfeã. quod ét vt faceré, iampridé in An glia rog atus erã a dńo Pratenfi pręfecto veftro, $g$ de publico ciuitatis hui ${ }^{9}$ bono, proinde 
Confulib' \& Senatui Brugéfi. proindeacdebet, multú \& fape cogitat." Vobis hocopusinfcribit.tum qpad benee faciendú \& fubleuãdosmiferos vehemét eftis propenfi. quod declarat tanta inopái

- multitudo, quę huc vndiqgaffluit tanğ ad paratú egenis fubfidiú .tum etiá op quă ea fit origo ciuitatú oium, vt effet vnaquę earumlocus, in quodandisatopaccipièdis beneficins \& $\&$ mutua fubuentione coalefce ret Charitas \& firmaret focietas hominu. adminiftratorum ciuitatisillud debet effe munus cusare \& anniti, vt alÿ al ̧̈s sauxis liofint. nemo prematur, nemo grauetur dáno per iniuraim accepto. \& imbecilliori adfit potentior. vt concordiacat ${ }^{9} \&$ cōgre $^{2}$ gationis ciuium charitate augefcat indies acfempiterna perfeueret. Et quéadmodú patrifamilię turpe êt in opuléta fua domo finere alique efurire; aut nuditate vel pan" nisfœedari,ficnöconuenit vt in vrbe haud pfus inopemagiftrat ${ }^{9}$ ferät ciues vllos far me $\&$ miferia vrgeri. Ne pigeat hæclege re.aut fi nō libet, certe rēip fă accuratiff.ex pédere. $q$ tã folliciticognofritis de lize hois priuati, in qua fint mille floréni controuer fi. opto vobis veftræç ciuitaci pfpera oía \& feticia. .M.D.XXVI.

Brugis.vi, lanuarij

$$
\text { A : i }
$$




\section{T R ATA D O D E L SOCORRO DE LOS POBRES COMPUESTO EN LATIN POR EL DOCTOR \\ JUAN LUIS VIVES \\ TRADUCIDO EN CASTELLANO P O R B L \\ Dr. JUAN DE GONZAZO, NIET O, IVARRA.}

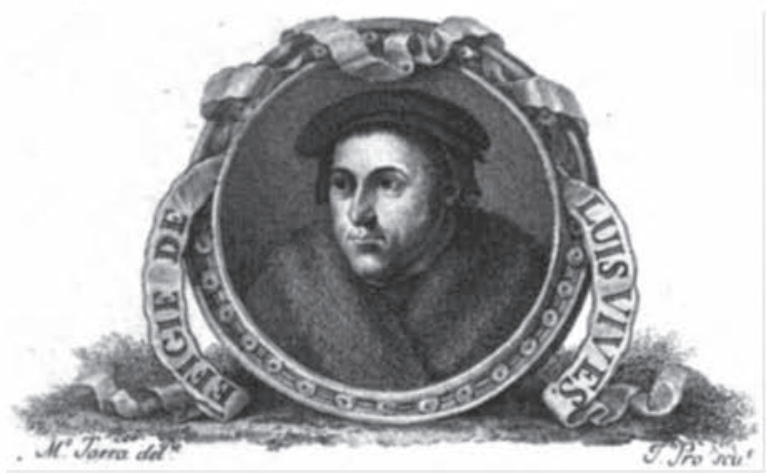

EN VALENCIA:

EN LA IMPRENTA DE BENITO MONFORT IMPRESOR DEL EX. ${ }^{\mathrm{mo}}$ S. ${ }^{\mathrm{r}}$ ARZOBıSPO, Año 1781 .

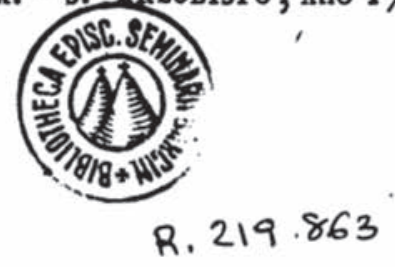

